\title{
ARTICLE Exploiting biased reptation for continuous flow preparative DNA fractionation in a versatile microfluidic platform
}

\author{
Burcu Gumuscu, Johan G. Bomer, Hans L. de Boer, Albert van den Berg and Jan C. T. Eijkel
}

A new approach is presented for preparative, continuous flow fractionation of sub-10-kbp DNA fragments, which exploits the variation in the field-dependent mobility of the DNA molecules based on their length. Orthogonally pulsed electric fields of significantly different magnitudes are applied to a microchip filled with a sieving matrix of $1.2 \%$ agarose gel. Using this method, we demonstrate a high-resolution separation of $0.5,1,2,5$, and $10 \mathrm{kbp}$ DNA fragments within 2 min. During the separation, DNA fragments are also purified from other ionic species. Preparative fractionation of sub-10-kbp DNA molecules plays an important role in second-generation sequencing. The presented device performs rapid high-resolution fractionation and it can be reliably manufactured with simple microfabrication procedures.

Keywords: agarose gel; biased reptation; continuous flow DNA separation; DNA purification; preparative fractionation

Microsystems \& Nanoengineering (2017) 3, 17001; doi:10.1038/micronano.2017.1; Published online: 22 May 2017

\section{INTRODUCTION}

Standard gel electrophoresis has been widely utilized for DNA fractionation in various genotyping and sequencing applications ${ }^{1-3}$. This method has the advantages of simplicity, versatility, and reproducibility. However, it suffers from long processing times on the order of a few tens of hours ${ }^{2,3}$. For instance, the Bio-Rad CHEF-DR device performs fractionation of 5-120 kbp DNA using pulsed-field gel electrophoresis (PFGE) in $25 \mathrm{~h}$ (Refs. 4,5). Trends toward second-generation sequencing motivate the replacement of standard gel electrophoresis with microchip-based systems, which could provide efficient platforms to minimize the processing time and to perform optimal DNA fractionation ${ }^{6-8}$.

Micro- and nanofabricated post arrays-that resemble a gel matrix with well-ordered and identical pores-have been integrated in microchip-based systems, increasing both the understanding of DNA separation principles and the fractionation's efficiency and speed. Two decades ago, Volkmuth and Austin $^{9}$ introduced a patterned micro-post array in a microfluidic electrophoresis platform, in which DNA fragments were separated by biased reptation under a DC electric field. Later, Duke et al. separated large fragments in a range of $60-135 \mathrm{kbp}$ in a microfabricated array using DNA reorientation-or the 'switchback' principle. Although the switchback principle is similar to what is used in PFGE, the device yields much faster separations owing to its sparse and regular sieving array ${ }^{10}$. In a later work, Kaji et al. studied a three-dimensional (3D) nanopost array as an optimal separation matrix for DNA fragments over a few kbps under a DC electric field, resulting in similarly fast separations due to the sparse matrix ${ }^{11}$.

With the aim of increasing the sample throughput and further facilitating the sample recovery, a continuous flow separation was developed. Huang et al. ${ }^{12}$ performed continuous flow PFGE in a micromachined post array by applying pulsed electric fields of slightly unequal strengths. DNA fragments ranging between 61 and $209 \mathrm{kbp}$ were separated within $15 \mathrm{~s}$ in the 'DNA prism'.
A comparable device was later incorporated into a gene analysis system and is currently being marketed by the company Pathogenix, to detect the genome of rare pathogenic bacteria from complex mixtures ${ }^{13}$. The operation time of this device is reported as $\sim 1.5 \mathrm{~h}$ to fractionate $60-200 \mathrm{kbp} \mathrm{DNA}^{13,14}$. Another reported variant of the 'DNA prism' device was a self-patterned 3D crystalline nanoarray used for fractionating smaller DNA molecules $(2-50 \mathrm{kbp})$ in a continuous flow setup ${ }^{15}$. Other separation principles, including Ogston sieving, entropic trapping, and electrostatic sieving, were demonstrated by $\mathrm{Fu}$ et al. using a nanofabricated two-dimensional (2D) sieving array operated under pulsed electric fields to fractionate DNA in a continuous flow ${ }^{16}$

The micro- and nanofabricated sieving devices briefly reviewed above allow for the optimization of the separation process by using spatially controlled sieving matrices that optimally exploit the basic physical principles of DNA separation. However, defectfree fabrication of the 3D nanostructures, such as the nanopost array ${ }^{11}$ and the crystalline nanoarray ${ }^{15}$, is extremely challenging. Although the manufacturing of 2D nanostructures (for example, the anisotropic nanofluidic sieving array ${ }^{16}$ ) is relatively easier, these nanostructures intrinsically yield low sample throughput. An ideal sieving matrix should thus have a simple design and facile fabrication steps yet provide high-resolution and high-throughput separation.

Remarkably, a relatively larger device (on the order of a few $\mathrm{cm}$ ) filled with bulk agarose gel has been demonstrated for the purification and concentration of high molecular weight DNA (on the order of tens of thousands of bp), using the field-dependent mobility of DNA in a method called synchronous coefficient of drag alteration (SCODA $)^{17-19}$. However, this study overlooked the fact that the field-dependent mobility depends on the DNA fragment size, which will be shown for the first time in this work to provide an alternative method for continuous flow DNA fractionation that has hitherto not been demonstrated. 
We demonstrate a microscale gel electrophoresis ( $\mu \mathrm{GEL}$ ) device operated under orthogonal pulsed electric fields with significantly differing magnitudes for continuous flow fractionation of sub-10-kbp DNA molecules. Preparative fractionation is shown here over a broad frequency range ( 3 decades). The $\mu \mathrm{GEL}$ device presents three major advances over the aforementioned microchip-based systems. First, agarose gel is used as the sieving matrix. This requires much less effort to fabricate a defect-free 3D network of nanopores for high-resolution separations, and offers considerable flexibility in varying the pore size for the DNA fragments of interest. Second, field-dependent mobility is explained and experimentally shown as a new separation mechanism for DNA fractionation. Third, we can combine both the separation and purification functions by maximally exploiting the field-dependent mobility. Fragments in a range of $0.5-10 \mathrm{kbp}$ are separated from each other and from other ionic species (fluorescein sodium salt was utilized in this work as an example) in a continuous flow.

\section{MATERIALS AND METHODS}

\section{Microchip fabrication}

The microchips were fabricated in the MESA+ cleanroom facility at the University of Twente. Fused silica glass was selected for its high optical clarity and its smooth microchannel surfaces after a reactive ion etching (DRIE) process. Microchips consisted of a processed top and an unprocessed bottom layers. The top layer contained the separation chamber, microchannels, and buffer reservoirs. A bonded microchip is presented in Figures $1 a$ and $b$, consisting of a 10 by $10 \mathrm{~mm}$ square chamber connected to the buffer reservoirs via microchannels $(50 \mu \mathrm{m} \times 10 \mathrm{~mm}, 50 \mu \mathrm{m}$ periodicity) on each side. The microchannels serve to generate a uniform electric field over the separation chamber. The overall dimension of the microchip is 35 by $35 \mathrm{~mm}$.

DRIE was utilized to define the microchannels and separation chamber and the process flow is shown in Figure 2. Before DRIE, the wafer was spin-coated with SU-8 (MicroChem), which served as a mask for the underlying surface. The SU-8 layer was patterned using photolithography and the residual SU-8 layer was stripped in a piranha bath after the DRIE process. The height of the structures was measured as $20 \mu \mathrm{m}$ using a Dektak 150 Surface Profiler (Veeco). Buffer reservoirs were opened on the back side of the top layer. After lamination and photolithography, the Ordyl foil resist (BF410, Ordyl) was developed using a $\mathrm{NaHCO}_{3}(0.2 \%$, w/v, Sigma Aldrich, Germany) solution. Subsequently, a powder blasting process was performed and the Ordyl foil was removed using acetone. Finally, both top and bottom layers were cleaned and thermally bonded at $1080^{\circ} \mathrm{C}$. The bonded wafers were then diced into individual microchips ${ }^{20}$.

Agarose hydrogel was prepared by dissolving agarose powder $(1.2 \%, w / v$, Invitrogen) in deionized water. The agarose gel with a $1.2 \%$ concentration was chosen because it yields the highest resolution separation for $0.5-10 \mathrm{kbp}$ DNA fragments in the microchip when compared to the agarose gels with $0.5,1$, and $1.5 \%$ concentrations. The mixture was boiled in a microwave and pipetted into the microchip that was warmed to $70^{\circ} \mathrm{C}$ on a hot plate. The empty space in the microchip was filled entirely by capillary forces ${ }^{20,21}$. The microchip was then immersed in a buffer solution overnight. The agarose gel expanded during the immersion process due to swelling, which ensured an airtight filling of the microchannels and eliminated leakage and air bubbles. The buffer solution consisted of tris borate EDTA (TBE, $0.1 \times$, Invitrogen), $\beta$-mercaptoethanol ( $3 \%, w / v$, Sigma Aldrich), and deionized water.

In our preliminary experiments, we used $0.5,1,1.2$, and $1.5 \%$ agarose concentrations to perform the separation. Among those experiments, we obtained the best resolution using the $1.2 \%$ agarose gel for the $0.5-10 \mathrm{kbp}$ fragments.

\section{Sample preparation}

Individual DNA fragments $(0.5,1,2,5$, and $10 \mathrm{kbp})$ were obtained from New England BioLabs. Fragments were labeled with an intercalating fluorescent dye YOYO-1 (Invitrogen) at a 1:5 dye-tobp ratio and the final DNA concentration was $12.5 \mathrm{ng} \mathrm{\mu L}^{-1}$. YOYO-1 solution was added before the DNA solution to prevent precipitation. The mixture was incubated at room temperature for $1 \mathrm{~h}$, and then $0.1 \%(\mathrm{v} / \mathrm{v}) \beta$-mercaptoethanol was added to prevent photo bleaching. The final mixture was stable at room temperature.

\section{Experiment setup}

An epifluorescence microscope (Leica, DM-IRM, Germany) equipped with a thermoelectrically cooled CCD camera (Hamamatsu

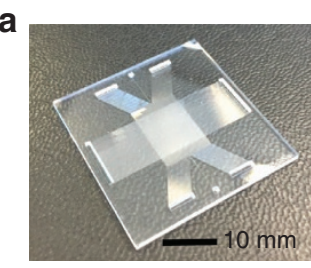

b

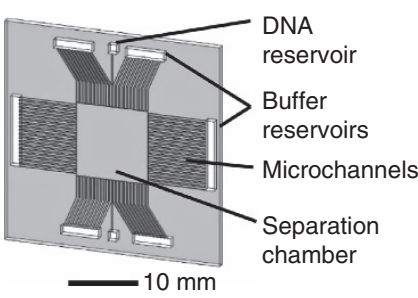

C

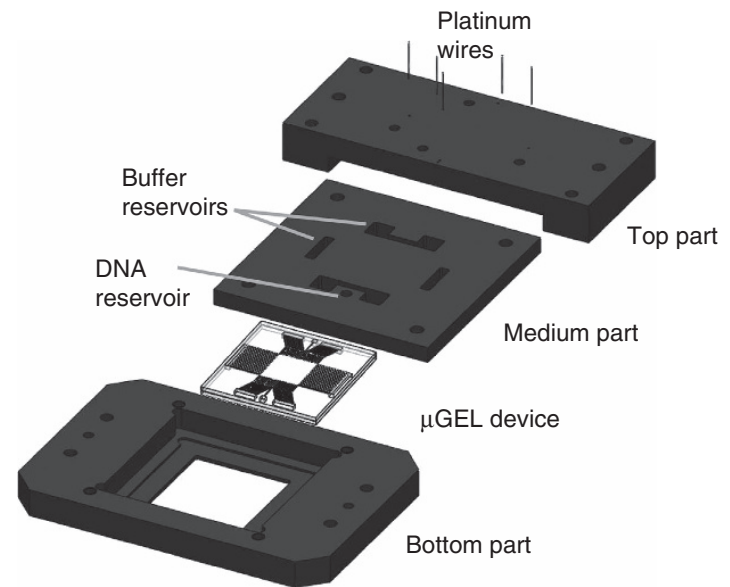

Figure 1 (a) An image of the fabricated microchip made of glass. (b) Schematic illustration of the microchip layout, including the DNA reservoir, buffer reservoirs, microchannels, and separation chamber. (c) Schematic illustration of the in-house made chip holder made of Delrin. The chip holder consists of three main parts. The bottom part has a window that enables the observation of DNA fractionation on an inverted epifluorescence microscope. The middle part has slits serving as the buffer reservoirs in direct contact with the buffer reservoirs on the microchip. The buffer reservoirs in the middle part are filled with buffer to enable contact with the platinum wires. The top part has holes to align the platinum wires to the reservoirs in the middle part. The wires are all connected to the power source. 


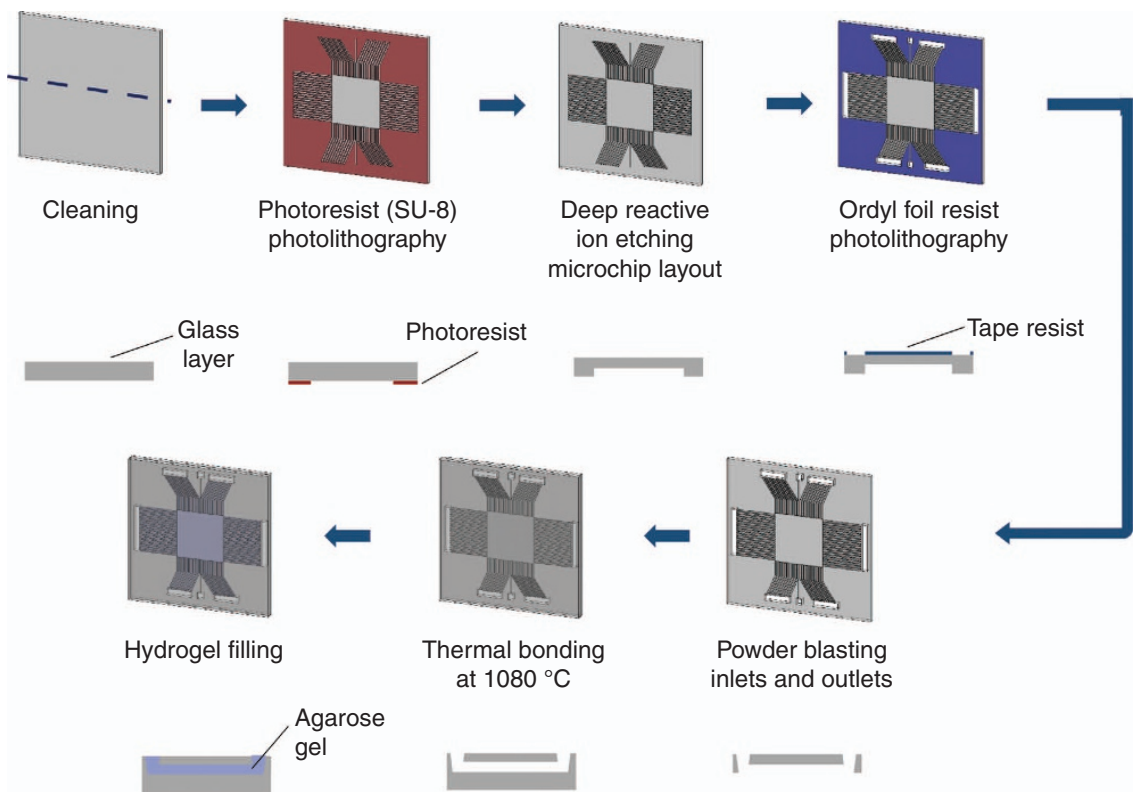

Figure 2 Schematic illustration of the microchip fabrication. The drawings at the bottom of the schematics present the cross sectional view along the blue dashed line in the top-left drawing. After the cleaning step, the layout of the microchip is defined by a photolithography step, which is followed by a reactive ion etching step. After this, the back side of the microchip is coated using Ordyl foil resist. The fluidic access holes are opened by performing photolithography and powder blasting steps, respectively. The processed layer is thermally bonded with an unprocessed layer to form microchannels. The microchip is finally filled with $1.2 \%$ agarose gel via capillary action.

ORCA-ER C4742-80-12AG) and GFP filter cube (Chroma) was used for fluorescence imaging. A $100 \mathrm{~W}$ mercury lamp (Leica) was set to the highest intensity level for illumination. Electric fields were applied to the microchip using a LabSmith HVS448LC High Voltage Sequencer (USA). In-house fabricated platinum electrode connectors were mounted in an in-house fabricated chip holder to apply the electric fields. Figure 1c illustrates the chip holder and connections.

\section{Simulations}

Two-dimensional finite element calculations of the $\mu \mathrm{GEL}$ device were performed to estimate the electric field distribution in the separation chamber, using the AC/DC module in the COMSOL Multiphysics 5.2 software in stationary conditions. The agarose gel and TBE buffer-filled separation chamber was modeled with a specific conductivity of $4.20 \mathrm{~S} \mathrm{~m}^{2}$ and a relative permittivity of 80 . The potentials applied to the reservoirs were defined as shown in Figure 3. The system responses to the applied potentials were governed by Maxwell's equations under the electric currents interface in the software.

\section{Resolution calculations}

We calculated the separation resolution between two adjacent flow streams $R_{\mathrm{s}}=\Delta X /\left(2 \sigma_{1}+2 \sigma_{2}\right)$, where $\Delta X$ is the spatial distance between the streams, and $\sigma_{1}$ and $\sigma_{2}$ are the standard deviations (s.d.) of the stream widths.

\section{Image processing}

Matlab software was used to plot the fluorescence intensity graphs. To obtain images with comparable intensities, the background of the images was subtracted, the noise was reduced by applying a Gaussian filter to every $5 \times 5$ group of pixels, and the contrast was increased by $2 \%$. The fluorescence intensity profile was plotted by drawing a line along the $y$ axis of the image (in front of the microchannels), where all the samples were collected. The resulting intensity plots were used to calculate the

\begin{tabular}{cccc}
\hline$E_{1}\left(\mathrm{~V} \mathrm{~cm}^{-1}\right)$ & $E_{2}\left(\mathrm{~V} \mathrm{~cm}^{-1}\right)$ & $\theta_{1}\left({ }^{\circ}\right)$ & $\theta_{2}\left({ }^{\circ}\right)$ \\
\hline 134.6 & 55.9 & 21.8 & 63.4 \\
89.8 & 34.4 & 21.8 & 76.0 \\
59.5 & 24.6 & 21.7 & 63.4 \\
29.1 & 9.7 & 18.0 & 67.8 \\
9.7 & 3.8 & 22.2 & 74.7 \\
\hline
\end{tabular}

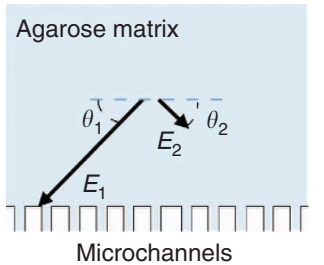

Figure 3 Magnitude of electric field strengths (left) and angles $\theta$ of the applied electric fields relative to the $x$ axis (right).

resolution of the separation. Resolution values between peaks are provided in Table 1.

\section{RESULTS AND DISCUSSION}

Due to the negative charge on their phosphate backbone, DNA molecules move against an applied electrical field as a result of Coulomb forces. DNA strands of different lengths can be separated by applying an electrical field in a porous gel matrix. The DNA velocity is thereby determined by both the electrical force and the size-dependent friction with the gel matrix. Three physical phenomena are crucial to understand the separation process described in this paper: the size dependence of the DNA mobility in the gel matrix, the field-strength dependence of the DNA mobility, and the time needed for the reorientation of the DNA molecules upon changes in the field direction (also called as 'switchback' mechanism). The size dependence of the mobility is caused by the tendency of DNA molecules to coil around the gel strands, which increases their friction. This tendency and increased friction increases with the DNA length. The field-strength dependence of the mobility stems from the tendency of the DNA molecules to align themselves in the field direction, which increases with increasing field strength. As a result, when the electric field direction is changed, the reorientation time depends 
Table 1 Peak purity of the collected streams in Figure 5b

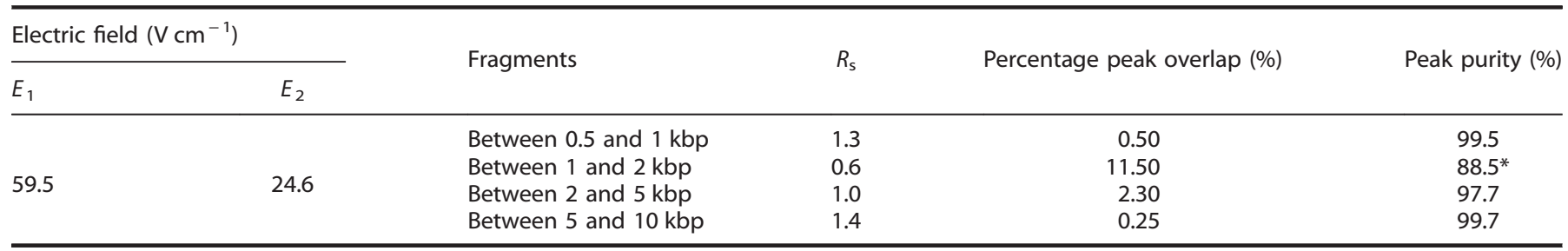

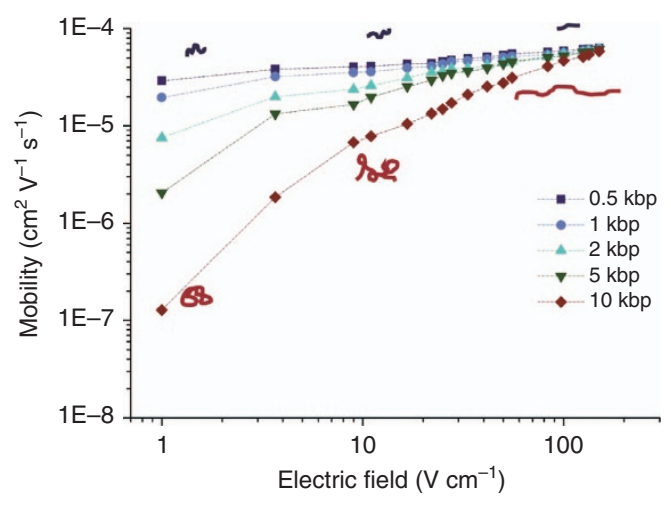

Figure 4 Measured mobility of individual DNA fragments as a function of the electric field. The red and dark blue figures illustrate the molecular conformation of large and small fragments at low and high electric fields, respectively.

on the DNA mobility and length. Figure 4 demonstrates both the size- and field-dependency of the mobility for different DNA lengths measured in the $\mu \mathrm{GEL}$ device. It will be demonstrated that by a combination of these properties, the $\mu \mathrm{GEL}$ device can separate DNA molecules of different lengths in continuous flow with a high resolution. This separation can be achieved by alternately applying high and low electric field strengths in orthogonal directions.

Figure 2 outlines the microchip fabrication and hydrogel patterning processes, while Figure 1 presents the design of the $\mu G E L$ device and the chip holder used in the experiments (see MATERIALS AND METHODS). The microchip consists of a $10 \mathrm{~mm}$ by $10 \mathrm{~mm}$ chamber connected to buffer reservoirs via parallel microchannel arrays $(50 \mu \mathrm{m} \times 10 \mathrm{~mm}, 50 \mu \mathrm{m}$ periodicity) on four sides. The microchannels provide a high-resistance area, preventing current leakage from the separation chamber and generating a fairly uniform electric field (Supplementary Figure $S 1)^{20,22}$.

In all separation experiments, two electric fields, $E_{1}$ and $E_{2}$, were alternately applied across the separation matrix at various frequencies. Figure 3 presents the magnitude and angle at the injection point-relative to the $x$ axis-for all the transverse electric fields. Throughout the experiments, the electric fieldstrength ratio $E_{1} / E_{2}$ at the injection point varied between 2.4 and 3 , while the angle between the fields varied around $\sim 90^{\circ}\left(85-98^{\circ}\right)$. The DNA fragments were continuously injected into the agarose matrix, and separated fragments were continuously collected at different sides of the microchannels.

Supplementary Figure S1 shows the simulated electric field distribution in the separation matrix at typical $E_{1}$ and $E_{2}$ values. Both the magnitude and direction of the electric fields vary slightly over the $\mu \mathrm{GEL}$ device. On approach of the collection point, the angle between $E_{1}$ and $E_{2}$ becomes more acute (up to $60^{\circ}$ at some locations in the microchip) while the ratio $E_{1} / E_{2}$ gradually increases (up to 3.1) as calculated using the COMSOL Multiphysics software.
We investigated the continuous flow separations of $0.5,1,2,5$, and $10 \mathrm{kbp}$ fragments, at a wide range of the DC electric fields $E_{1}$ and $E_{2}$ from 1 to $135 \mathrm{~V} \mathrm{~cm}^{-1}$ and switching frequencies from 0.016 to $33 \mathrm{~Hz}$. Figure 5 shows photomicrographs of the flow streams of the individual DNA fragments observed at the various electric fields and switching frequencies. The deflection angle $(\Phi)$ between the initial stream and the flow stream exit locations was measured to quantify the separation between the fragments (Figure 6f).

The semi-log plots of the deflection angle as a function of the switching frequency, given in Figures $6 a-e$, show that the DNA fragments can be separated over more than three decades of frequency. To explain this observed behavior, we propose a mechanism based on a combination of the field-dependent mobility and the 'switchback' mechanisms, with the former mechanism dominating at low frequencies and the latter mechanism dominating at higher frequencies. The switchback mechanism was previously demonstrated in micromachined devices by Huang et $a l^{12}$ and Zeng et $a l^{15}{ }^{15}$, and theoretically analyzed by Chen et $a l^{23}$. When the applied field switches direction, the DNA fragments need time to reorient, $t_{\mathrm{or}} \approx \frac{L}{\mu E}$, where $L$ is the DNA contour length, $\mu$ the DNA mobility, and $E$ is the applied field ${ }^{19}$. This reorientation time is shorter in the large field $E_{1}$ than in the small field $E_{2}$. The corresponding reorientation frequencies are $f_{\text {or }, 1}=\frac{1}{2 t_{\text {or }, 1}} \approx \frac{\mu E_{1}}{2 L_{\mathrm{DNA}}}$ and $f_{\mathrm{or}, 2}=\frac{1}{2 t_{\mathrm{or}, 2}} \approx \frac{\mu E_{2}}{2 L_{\mathrm{DNA}}}$. At a switching frequency below $f_{\text {or }, 1}$ and above $f_{\text {or }, 2}$, DNA molecules can only reorient in the strong field $\left(E_{1}\right)$ and will follow the field vector of this field. At frequencies below $f_{\text {or, } 2}$ or above $f_{\text {or, }, 1}$ DNA will follow an intermediate trajectory. As the reorientation frequencies are also a function of the DNA length, the migration angle at frequencies $\frac{\mu E_{2}}{2 L_{D N A}}<f<\frac{\mu E_{1}}{2 L_{D N A}}$ depends on the DNA length $L_{D N A}$, enabling separation around these frequencies. In our system the fractionation at these frequencies can be partially explained by the switchback mechanism. In Figures $6 a-e$, the frequencies around and above these values are indicated with a purple background.

The physics of DNA reorientation in agarose gels was studied by Aakerman et al. ${ }^{24}$ The reorientation frequencies of DNA fragments in the $\mu \mathrm{GEL}$ device lie between $2.4 \mathrm{~Hz}$ for $10 \mathrm{kbp}$ fragments and $50 \mathrm{~Hz}$ for $0.5 \mathrm{kbp}$ fragments at an applied field of $59.5 \mathrm{~V} \mathrm{~cm}^{-1}$. Optical measurements in the $\mu \mathrm{GEL}$ device confirmed the validity of the equation $f_{\text {or }}=\frac{1}{2 t_{\text {or }}} \approx \frac{\mu E}{2 L_{D N A}}$ for the switching frequency (Supplementary Figure S3). Calculated orientation times for the 10 -kbp fragments were $0.24 \mathrm{~s}$ when $E_{1}$ was switched to $E_{2}$, and $0.42 \mathrm{~s}$ when $E_{2}$ was switched to $E_{1}$, when the magnitudes were $59.5 \mathrm{~V} \mathrm{~cm}^{-1}\left(E_{1}\right)$ and $24.6 \mathrm{~V} \mathrm{~cm}^{-1}\left(E_{2}\right)$. The observed reorientation times were found to be in good agreement with theory, calculated as 0.2 and $0.4 \mathrm{~s}$, respectively. At frequencies far below $f_{\mathrm{or}, 2}=\frac{1}{2 t_{\mathrm{or}, 2}} \approx \frac{\mu E_{2}}{2 L_{\mathrm{DNA}}}$, the DNA fragments will quickly reorient along the new electric field direction and will spend most of the application time moving in steady state along the field ${ }^{20}$. Different from the predictions of the switchback mechanism, we still observe separation at these frequencies. The fractionation observed at these frequencies can now be explained by the variation in the field-dependent mobility of the DNA molecules based on their length. The biased reptation with the orientation 

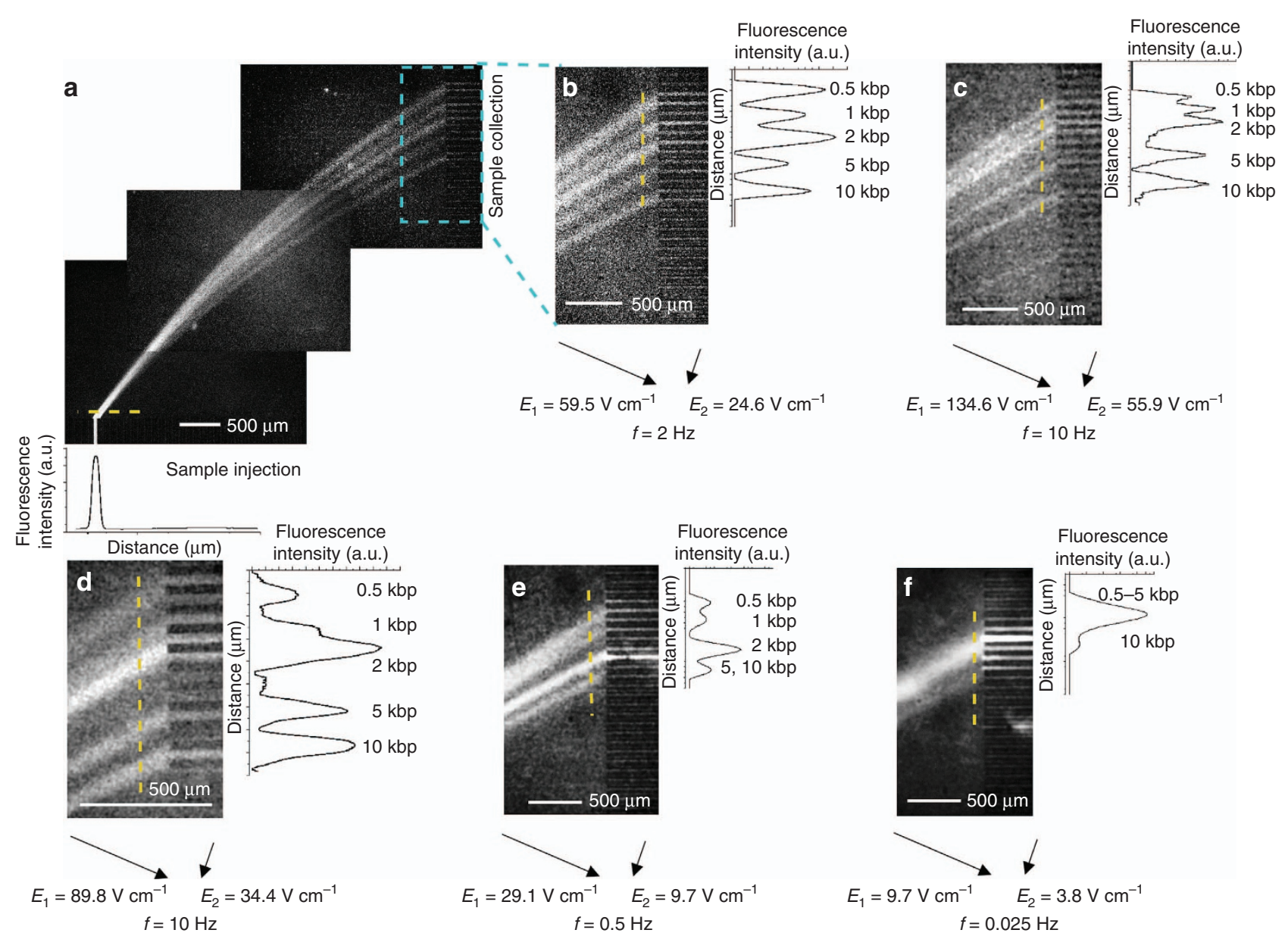

Figure 5 DNA fractionation in a continuous flow. (a-f) Fluorescence images of the fractionating 0.5-10 kbp DNA in an agarose sieving matrix. The intensity profiles (right) were obtained from the fluorescent images (left) by scanning over the yellow dashed line. Each image was recorded with a $12 \mathrm{~s}$ exposure time.

principle states that the mobility, $\mu$, of a DNA fragment in an agarose sieving matrix is a function of the electric field and DNA contour length ${ }^{23,25-28}$. This behavior was experimentally confirmed in the $\mu \mathrm{GEL}$ device. Figure 4 shows the measured fielddependent mobilities. At low frequencies, the reorientation time can be neglected and the migration trajectories can simply be calculated by adding the trajectories at the high $\left(E_{1}\right)$ and low $\left(E_{2}\right)$ fields (Figure 6f). The larger the DNA fragment, the larger the mobility increases between the two applied fields $\Delta \mu=\mathrm{d} \mu / \mathrm{d} E^{*}$ $\left(E_{1}-E_{2}\right)$ (Figures 4 and $6 f$ ), leading to fragments of different sizes following different trajectories.

Adding the contributions of both separation mechanisms, the migration angle of the DNA molecules with respect to the horizontal axis, $\Phi$, can be approximated by the following equation:

$$
\Phi=\operatorname{atan}\left(\frac{\sin \theta_{1}+\left(\frac{E_{2} \mu_{2}-2 f L}{E_{1} \mu_{1}-2 f L}\right) \sin \theta_{2}}{\cos \theta_{1}-\left(\frac{E_{2} \mu_{2}-2 f L}{E_{1} \mu_{1}-2 f L}\right) \cos \theta_{2}}\right)
$$

Here $\mu_{1,2}$ is the mobility of the fragments at the two applied fields $E_{1,2} ; \theta_{1,2}$ is the angle between the transverse electric fields, $E_{1}$ or $E_{2}$, and the $x$ axis; $f$ the applied frequency; and $L$ is the DNA length.

The calculated (Equation (1)) and measured deflection angles of the fragments for the $59.5 \mathrm{~V} \mathrm{~cm}^{-1}\left(E_{1}\right)$ and $22.4 \mathrm{~V} \mathrm{~cm}^{-1}\left(E_{2}\right)$ fields are presented in the Supplementary Figure S2. Comparing S2a and S2b shows that four flow streams are obtained at all the frequencies below $1 \mathrm{~Hz}$ (Figures $5 \mathrm{a}$ and $6 \mathrm{c}$ ). This finding corresponds to the separation of the $0.5-5 \mathrm{kbp}$ fragments and can be attributed to the biased reptation mechanism. Because the 5 and $10 \mathrm{kbp}$ fragments have approximately equal mobilities $\left(\mu_{1} / \mu_{2}\right)$ at $E_{1}$ and $E_{2}$, they cannot be separated by the biased reptation mechanism. However, we obtained five flow streams in the frequency range from 0.5 to $10 \mathrm{~Hz}$ (Figures $5 \mathrm{a}$ and $6 \mathrm{c}$ ) with $>1$ resolution between 2 and $10 \mathrm{kbp}$ fragments. For the 5 and $10 \mathrm{kbp}$ fragments, we expect that the reorientation mechanism contributes to the separation because the reorientation frequencies of these DNA lengths are 2 and $4 \mathrm{~Hz}$, respectively. It was furthermore found that the calculated angles were lower than the measured angles. This difference can be explained by (1) the bent electric field lines and the variation in field magnitude throughout the microchip, which leads to larger than predicted angles (Supplementary Figure S1); and (2) the fact that the separated flow streams fluctuated along the $y$ axis at low frequencies, leading to an increased uncertainty in the angle determination. A rough correction for the curved field lines can be based on the measured angle of the fluorescein sodium salt trajectory (Supplementary Video S5), for which $\mu_{1} / \mu_{2}=1$.

According to the field-dependent mobility mechanism, the best separation performance for all the DNA lengths is expected when $\mu_{1} / \mu_{2}$ varies maximally for all DNA lengths. According to Figure 6 , the best separation performance should be achieved around $E_{2} \approx 25 \mathrm{~V} \mathrm{~cm}^{-1}$. In our observations, the highest resolution separation was indeed obtained within this field region, specifically when applying $59.5 \mathrm{~V} \mathrm{~cm}^{-1}\left(E_{1}\right)$ and $22.4 \mathrm{~V} \mathrm{~cm}^{-1}\left(E_{2}\right)$. 

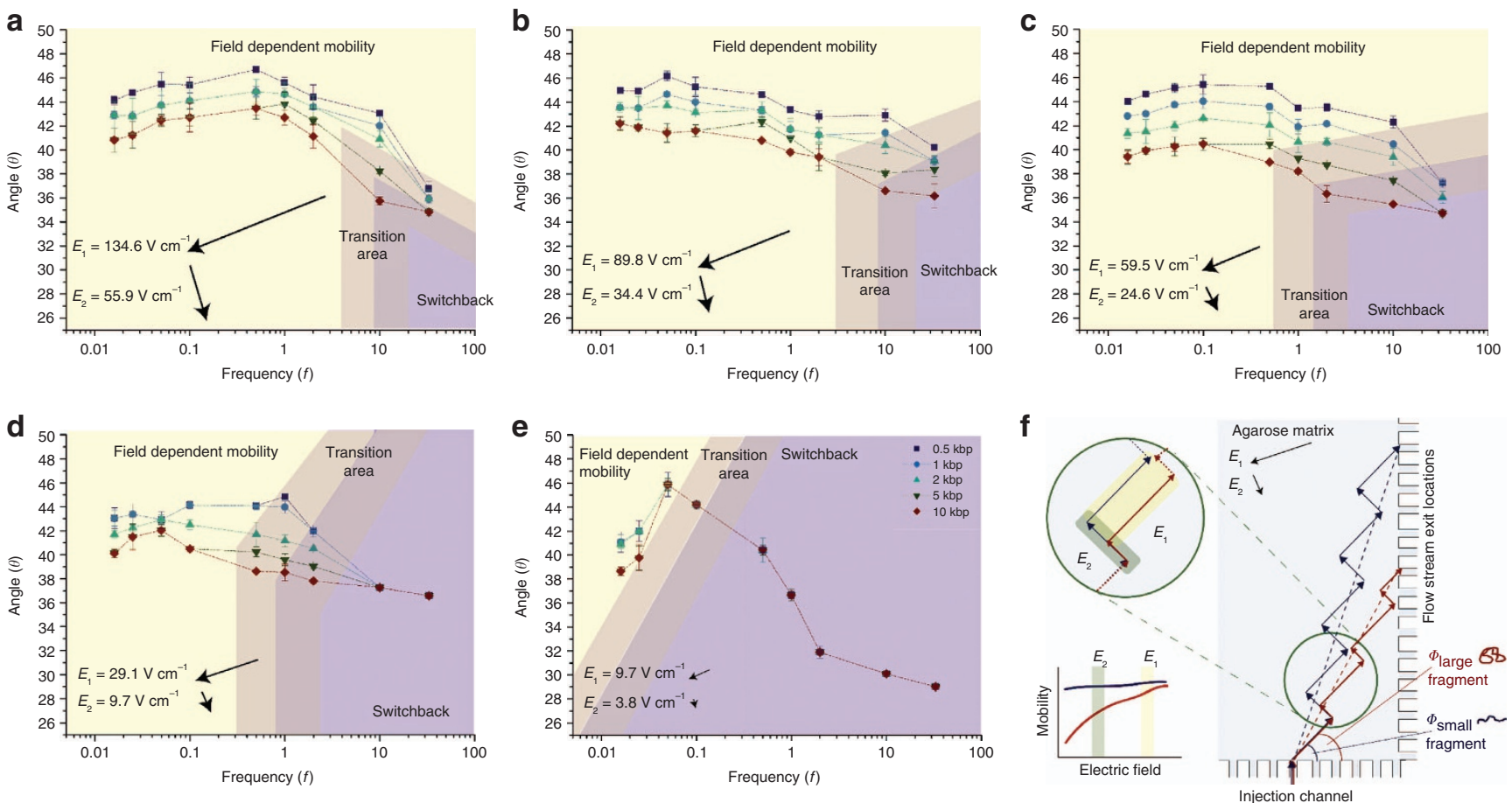

Figure 6 (a-e) Frequency spectra of the deflection angle $\theta$ for various DNA fragments under different field strengths. Error bars show s.d. $(n=2)$. (f) Schematic representation of the separation principle at high electric field strength and low frequency. Blue and red dashed lines present the net migration trajectory of each fragment. $\Phi$ is the deflection angle of the DNA fragments.

Figure $5 \mathrm{c}$ shows that five flow streams with good resolution were obtained when applying $134.6 \mathrm{~V} \mathrm{~cm}^{-1}\left(E_{1}\right)$ at $10 \mathrm{~Hz}$ (the reorientation frequency of the DNA fragments in this field is $100 \mathrm{~Hz}$ for $0.5 \mathrm{kbp}$ DNA and $5 \mathrm{~Hz}$ for $10 \mathrm{kbp}$ DNA). At this frequency, both the field-dependent mobility (for small fragments) and switchback mechanisms (for large fragments) are expected to contribute to the resolution.

At low-frequency ranges $(0.05-10 \mathrm{~Hz})$ and an $89.8 \mathrm{~V} \mathrm{~cm}^{-1} E_{1}$, separation was consistently obtained for four flow streams, as seen in Figures $5 \mathrm{~d}$ and $6 \mathrm{~b}\left(6 \mathrm{~Hz}<f_{\text {or }}=120 \mathrm{~Hz}\right.$ for the DNA fragments). As another example, applying $29.1 \mathrm{~V} \mathrm{~cm}^{-1}\left(E_{1}\right)$ at a $1 \mathrm{~Hz}$ frequency yielded a satisfactory separation $\left(R_{\mathrm{s} \text { mean }} \approx 0.7\right)$ with four independent flow streams (Figure 6d). At this field, $2 \mathrm{~Hz}<f_{\text {or }}<40 \mathrm{~Hz}$ for the DNA fragments, indicating that the field-dependent mobility is the major contributor to the separation. As seen in Figure 6e, the switchback mechanism does not work well in the $\mu \mathrm{GEL}$ device when operated under low electric field strengths as the separation performance decreased when $f_{\text {or }}<0.1 \mathrm{~Hz}$. The poorest separation was obtained when $9.7 \mathrm{~V} \mathrm{~cm}^{-1}\left(E_{1}\right)$ was applied at the lowest frequency, $0.016 \mathrm{~Hz}$. In this case, only two flow streams of fragments were separated.

The field-dependent mobility mechanism theoretically yields an angle $\Phi$ independent of the frequency. Figures $6 a-e$ show this independence only at low frequencies. The observed decrease in angle with an increase in frequency is most likely due to the reorientation mechanism. For example, 5 and $10 \mathrm{kbp}$ fragments were separated at $59.5 \mathrm{~V} \mathrm{~cm}^{-1}\left(E_{1}\right)$ and $22.4 \mathrm{~V} \mathrm{~cm}^{-1}$ $\left(E_{2}\right)$ owing to the decreasing angle with increasing frequency. The agarose gel does not appear to yield satisfactory separations in the switching regime using the applied voltage protocol, despite the resolution decreasing for all field strengths at higher frequencies.

Throughout the experiments, the angle between $E_{1}$ and $E_{2}$ varied by $\sim 90^{\circ}\left(85-98^{\circ}\right)$, which decreased to an angle of $60^{\circ}$ at the exit location of the flow streams. In PFGE (where the reorientation mechanism is used), the best separation performances were always obtained when the angle between the two equal transverse electric fields was $120^{\circ}$ (not $\left.90^{\circ}\right)^{24}$. However, in the case of the fielddependent mobility mechanism, the migration velocity differences determine the separation process. We optimally exploited the velocity differences with the applied orthogonal fields.

Up to 80 different experiments could be performed with the same microchip, using a large variety of voltage and frequency protocols, and yielding similar (>95\%) results between the replicates of the experiments. Figure 6 shows the s.d. of the measurements performed using the same microchip. Different microchips could also be used for fractionation and purification with reproducible results ( $>70 \%)$.

For the DNA recovery calculations, we integrated the fluorescence over the width of the flow stream, both at the inlet and the outlet, and compared the results after subtracting the baseline intensity. For example, upon the alternating application of $59.5 \mathrm{~V} \mathrm{~cm}^{-1}\left(E_{1}\right)$ and $24.6 \mathrm{~V} \mathrm{~cm}^{-1}\left(E_{2}\right)$ fields at a $2 \mathrm{~Hz}$ frequency where the highest resolution was observed, the integrated fluorescence over the width of the flow stream at the inlet was 2897 (dimensionless number), and at the outlet (sum of all fragment streams) 2783 (dimensionless number). This corresponds to a recovery of $96.06 \%$, which is as good as previously reported sequencing devices ${ }^{29,30}$.

The peak purity of the fragment streams was calculated using our resolution values by assuming Gaussian peak shapes. According to this analysis, the purity of the collected streams in Figure $5 b$ are shown in Table 1.

Due to the low-height $(20 \mu \mathrm{m})$ channels and separation chamber, Joule heating is not expected to contribute significantly to the separations in the applied field range. According to Supplementary Figure S4, Joule heating might have affected the separation performance at the highest field strengths $\left(E_{1}=134.6 \mathrm{~V} \mathrm{~cm}{ }^{-1}\right)$. The ionic strength of the buffer solution was kept constant throughout the experiments. The reservoir volume is 2000 times larger than the inner volume of the microchip and the buffer solution was frequently refreshed. 
The $\mu$ GEL device has a calculated throughput of $0.18 \mathrm{ng}$ of molecules per hour at the DNA input concentration of $12.5 \mathrm{ng} \mathrm{\mu L}^{-1}$ and is comparable to previously reported microfabricated devices ${ }^{20,31,32}$.

In our experiments, we found that the width of the band at the injection site $(110 \mu \mathrm{m})$ did not significantly differ from the width of the bands at the flow stream exit locations (120-200 $\mu \mathrm{m})$. Thus, band broadening was minimal during the separation process, which is in accordance with the results suggested by Huang et $a .^{12}$ and Lerch et al. ${ }^{33}$ At low frequencies, however, the sawtooth movement of the DNA fragments is a major contributor to band broadening. The contribution of the alternating field protocol can be quantified by

$$
L_{\mathrm{fr}}=\frac{0.5 \mu_{2} E_{2}}{f}\left(\tan \theta_{1} \cos \theta_{2}+\sin \theta_{2}\right)
$$

where $L_{\mathrm{fr}}$ is the distance along the vertical axis travelled by a DNA fragment in one switching period. According to Equation (2), the contribution of the field switching (that is, fluctuation of fragment flow streams along the separation matrix) to the total band broadening will be less than $30 \%(\sim 33 \mu \mathrm{m}$ band width) when $f<0.05 \mathrm{~Hz}$ and $E_{2}=24.6 \mathrm{~V} \mathrm{~cm}^{-1}$. Because the width of the injection band is the main contributor to the band variance, the resolution of the $\mu \mathrm{GEL}$ device could be improved by decreasing the injection channel width.

The peak width variances between the injection channel and flow stream exit locations at $>10 \mathrm{~Hz}$ and $>2 \mathrm{~Hz}$ are $<5 \%$ and $15 \%$, respectively. Peak width variations are given in Figure 5.

For other ionic species, no reptation occurs and the molecules have the same mobility under both electric fields. Thus, the $2 f L$ term drops and $\mu_{2}=\mu_{1}$, reducing Equation (1) to

$$
\Phi=\operatorname{atan}\left(\frac{E_{1} \sin \theta_{1}+E_{2} \sin \theta_{2}}{E_{1} \cos \theta_{1}+E_{2} \cos \theta_{2}}\right)
$$

Periodic application of $E_{1}$ and $E_{2}$ results in the ionic species following a path at an angle $\Phi_{0}>\Phi$ (Supplementary Video S5) ${ }^{27}$. When the applied field strengths were $59.5 \mathrm{~V} \mathrm{~cm}^{-1}\left(E_{1}\right)$ and $22.4 \mathrm{~V} \mathrm{~cm}^{-1}\left(E_{2}\right)$, we calculated $\Phi_{0}=45^{\circ}$ for the fluorescein sodium salt, predicting clear separation from the DNA fragments with a calculated $\Phi<38.5^{\circ}$ (Supplementary Figure S2). We experimentally confirmed the purification by separating a mixture of 0.5-10 kbp fragments and $10 \mathrm{mM}$ fluorescein sodium salt by applying $59.5 \mathrm{~V} \mathrm{~cm}^{-1}\left(E_{1}\right)$ and $22.4 \mathrm{~V} \mathrm{~cm}^{-1}\left(E_{2}\right)$ at $2 \mathrm{~Hz}$ (Supplementary Video S5).

The advantages of the proposed device include its simple design and fabrication process. Here we used the device to separate sub-10 kbp DNA fragments. However, the use of agarose gel as the sieving matrix provides flexibility, which in principle enables fractionation of DNA fragment sizes outside the $0.5-10 \mathrm{kbp}$ range studied here by changing the gel concentration. A similar strategy is used in the commercially available mate-pair sequencing device, BluePippin (SageElf Inc.), which performs capillary electrophoresis for DNA fractionation in a precast agarose gel cassette with orthogonal sample plug extraction wells ${ }^{34}$. BluePippin can process between a few tens to thousands of base pairs, simply by changing the agarose concentration and voltage protocol. However, fractionation of sub-10 kbp DNA fragments requires $4 \mathrm{~h}$ using BluePippin ${ }^{9}$, while the $\mu \mathrm{GEL}$ device presented here requires only $2 \mathrm{~min}$.

The band resolution for 0.5 to $10 \mathrm{kbp}$ fragments and fluorescein sodium salt varied between 0.9 and 1.4 , when 59.5 and $24.6 \mathrm{~V} \mathrm{~cm}^{-1}$ fields were switched at a frequency of $2 \mathrm{~Hz}$. Because the main contributor to the band variance was the injection band width, we expect that resolution in the $\mu \mathrm{GEL}$ device can be further improved by decreasing the width of the injected band. Improvements in the band separation will be attempted by optimizing the voltage protocol to separate the fragment sizes. Chen et al. ${ }^{23}$ predicted that large and small fragments at low frequencies $\left(<2 / t_{\text {or }}\right)$ will migrate through isotropic matrices such as agarose gels at similar angles and no continuous flow fractionation would be possible. However, $\mu_{1} / \mu_{2}$ was assumed to be constant by Chen et al., which is incorrect. As a result, we could achieve fractionation using the fielddependent mobility of DNA.

\section{CONCLUSIONS}

We have introduced a novel microfluidic platform for the purification and high-resolution fractionation of DNA molecules. A new separation principle is explained based on variations in the field-dependent mobility of DNA molecules with respect to their length. The use of the agarose separation matrix enabled simple, fast, and extremely low-cost fabrication. This device would be of a broad interest for second-generation sequencing and clinical diagnosis applications, as it can achieve similar performance with much less effort in terms of fabrication and operation in comparison with currently available devices. In addition, the $\mu \mathrm{GEL}$ device can further be extended to protein gel electrophoresis by replacing the agarose gel with a polyacrylamide gel ${ }^{35}$.

\section{ACKNOWLEDGEMENTS}

This work was funded by the Dutch network for Nanotechnology, NanoNext NL, in the subprogram 'Nanofluidics for Lab-on-a-chip'. We thank Jan W. van Nieuwkasteele and Ad Sprenkels for technical support, Loes I. Segerink and Hai Le The for their kind help in image processing, and Allison C.E. Bidulock and Joshua Loessberg-Zahl for thorough discussions.

\section{COMPETING INTERESTS}

The authors declare no conflict of interest.

\section{REFERENCES}

1 Carle GF, Frank M, Olson MV. Electrophoretic separations of large DNA molecules by periodic inversion of the electric field. Science 1986; 232: 65-68.

2 Slater GW, Mayer P, Drouin G. Migration of DNA through gels. Methods in Enzymology 1996; 270: 272-295.

3 Tautz D, Renz M. An optimized freeze-squeeze method for the recovery of DNA fragments from agarose gels. Analytical Biochemistry 1983; 132: 14-19.

4 Lin YT, Labbe R. Enterotoxigenicity and genetic relatedness of Clostridium perfringens isolates from retail foods in the United States. Applied and Environmental Microbiology 2003; 69: 1642-1646.

5 Kwan HS, Li CC, Chiu SW et al. A simple method to prepare intact yeast chromosomal DNA for pulsed field gel electrophoresis. Nucleic Acids Research 1991; 19: 1347.

6 Han J, Turner SW, Craighead HG. Entropic trapping and escape of long DNA molecules at submicron size constriction. Physical Review Letters 1999; 83: 1688.

7 Han J, Craighead HG. Separation of long DNA molecules in a microfabricated entropic trap array. Science 2000; 288: 1026-1029.

8 Meistermann L, Tinland B. DNA electrophoresis in a monodisperse porous medium. Physical Review E 2000; 62: 4014.

9 Volkmuth WD, Austin RH. DNA electrophoresis in microlithographic arrays. Nature 1992; 358: 600-602.

10 Duke TA, Austin RH, Cox EC et al. Pulsed-field electrophoresis in microlithographic arrays. Electrophoresis 1996; 17: 1075-1079.

11 Kaji N, Tezuka Y, Takamura Y et al. Separation of long DNA molecules by quartz nanopillar chips under a direct current electric field. Analytical Chemistry 2004; 76: 15-22.

12 Huang LR, Tegenfeldt JO, Kraeft JJ et al. A DNA prism for high-speed continuous fractionation of large DNA molecules. Nature Biotechnology 2002; 20: 1048-1051.

13 Meltzer RH, Krogmeier JR, Kwok LW et al. A lab-on-chip for biothreat detection using single-molecule DNA mapping. Lab on a Chip 2011; 11: 863-873.

14 Kloke A, Fiebach AR, Zhang S et al. The LabTube-a novel microfluidic platform for assay automation in laboratory centrifuges. Lab on a Chip 2014; 14: 1527-1537.

15 Zeng Y, He M, Harrison DJ. Microfluidic self-patterning of large-scale crystalline nanoarrays for high throughput continuous DNA fractionation. Angewandte Chemie 2008; 120: 6488-6491.

$16 \mathrm{Fu} \mathrm{J,} \mathrm{Schoch} \mathrm{RB,} \mathrm{Stevens} \mathrm{AL} \mathrm{et} \mathrm{al.} \mathrm{A} \mathrm{patterned} \mathrm{anisotropic} \mathrm{nanofluidic}$ sieving structure for continuous-flow separation of DNA and proteins. Nature Nanotechnology 2007; 2: 121-128. 
17 Pel J, Broemeling D, Mai L et al. Nonlinear electrophoretic response yields a unique parameter for separation of biomolecules. Proceedings of the National Academy of Sciences of United States of America 2009; 106: 14796-14801.

18 Marziali A, Pel J, Bizzotto D et al. Novel electrophoresis mechanism based on synchronous alternating drag perturbation. Electrophoresis 2005; 26: 82-90.

19 Frumin LL, Peltek SE, Zilberstein GV. Nonlinear focusing of DNA macromolecules. Physical Review E 2001; 64: 021902.

20 Gumuscu B, Bomer JG, van den Berg A et al. Large scale patterning of hydrogel microarrays using capillary pinning. Lab on a Chip 2015; 15: 664-667.

21 Gumuscu B, Bomer JG, van den Berg A et al. Photopatterning of hydrogel microarrays in closed microchips. Biomacromolecules 2015; 16: 3802-3810.

22 Lerch MA, Jacobson SC. Electrokinetic fluid control in two-dimensional planar microfluidic devices. Analytical Chemistry 2007; 79: 7485-7491.

23 Chen Z, Dorfman KD. Relationship between frequency and deflection angle in the DNA prism. Physical Review E 2013; 87: 012723.

24 Aakerman B, Jonsson M. Reorientational dynamics and mobility of DNA during pulsed-field agarose gel electrophoresis. Journal of Physical Chemistry 1990; 94: 3828.

25 Viovy JL. Electrophoresis of DNA and other polyelectrolytes: Physical mechanisms. Reviews of Modern Physics 2000; 72: 813.

26 Stellwagen NC. Apparent pore size of polyacrylamide gels: Comparison of gels cast and run in Tris-acetate-EDTA and Tris-borate-EDTA buffers. Electrophoresis 1998; 19: 1542-1547.

27 Dorfman KD, King SB, Olson DW et al. Beyond gel electrophoresis: Microfluidic separations, fluorescence burst analysis, and DNA stretching. Chemical Reviews 2012; 113: 2584-2667.

28 Viovy JL. Reptation-Breathing theory of pulsed electrophoresis: Dynamic regimes, antiresonance and symmetry breakdown effects. Electrophoresis 1989; 10: 429-441.
29 Huang T, Shen P, Shen Y. Preparative separation and purification of deoxyschisandrin and $\gamma$-schisandrin from Schisandra chinensis (Turcz.) Baill by high-speed counter-current chromatography. Journal of Chromatography A 2005; 1066: 239-242.

30 Papadoyannis IN, Gika HG. Peak purity determination with a diode array detector. Journal of Liquid Chromatography \& Related Technologies 2004; 27: 1083-1092.

31 Tabuchi M, Ueda M, Kaji N et al. Nanospheres for DNA separation chips. Nature Biotechnology 2004; 22: 337-340.

32 Yasui T, Rahong S, Motoyama $\mathrm{K}$ et al. DNA manipulation and separation in sublithographic-scale nanowire array. ACS Nano 2013; 7: 3029-3035.

33 Lerch MA, Hoffman MD, Jacobson SC. Influence of channel position on sample confinement in two-dimensional planar microfluidic devices. Lab on a Chip 2008; 8: 316-322. 34 Heavens D, Accinelli GG, Clavijo B et al. Benchmarks. BioTechniques 2015; 59: 42-45.

35 Huang LR, Tegenfeldt JO, Kraeft JJ et al. Generation of large-area tunable uniform electric fields in microfluidic arrays for rapid DNA separation. International Electron Devices Meeting, 2001 (IEDM '01) Technical Digest; 2-5 Dec 2001; Washington DC, USA; 2001: 363.

This work is licensed under a Creative Commons Attribution 4.0 International License. The images or other third party material in this article are included in the article's Creative Commons license, unless indicated otherwise in the credit line; if the material is not included under the Creative Commons license, users will need to obtain permission from the license holder to reproduce the material. To view a copy of this license, visit http://creativecommons.org/licenses/ by/4.0/

(c) The Author(s) 2017

Supplementary Information for this article can be found on the Microsystems \& Nanoengineering website (http://www.nature.com/ micronano) 